\title{
Deformation of a lipid vesicle in an electric field: a theoretical study
}

\author{
A. Sokirko ${ }^{1 \star}$, V. Pastushenko ${ }^{2 \star \star}$, S. Svetina ${ }^{3}$ and B. Žekš ${ }^{3}$ \\ ${ }^{\prime}$ Department of Chemistry, Trent University, Peterborough K9J 7BR Ontario (Canada) \\ Institute of Biophysics, J. Kepler University of Linz, Altenbergerstr. 69, A-4040 Linz-Auhof (Austria) \\ ${ }^{3}$ Institute of Biophysics, Medical Faculty and J. Stefan Institute, University of Ljubljana, Lipičeı a 2, 61105 Ljubljana (Slovenia)
}

(Received 3 January 1994: in revised form 4 March 1994)

\begin{abstract}
The deformation of a lipid vesicle in an external electric field is analysed assuming constant membrane area and variable vesicle volume due to water transport through the membrane. The shape of the vesicle is approximated by a rotational ellipsoid. The equilibrium ratio of the semi-axes varies monotonically with field strength $\mathrm{E}$, ,. This ratio is useful for experimental determination of the bending elasticity modulus. The kinetics of vesicle deformation is described by the characteristic relaxation time $\tau$. This time increases drastically with vesicle size. A non-monotonic dependence of $\tau$ on $\mathrm{E}_{0}$ is shown numerically and analytically. For weak and strong fields $\tau$ is proportional to $E_{0}^{4}$ and $E_{11}^{-8 / 3}$ respectively.
\end{abstract}

\section{Introduction}

Application of an electric field causes deformation of lipid vesicles and cells. Deformation of lipid vesicles as well as of cells with no internal structure (such as erythrocytes), depends on the elastic properties of their membranes. Therefore studies of the dependence of a vesicle or a cell deformation on the strength of the electric field enable the elastic parameters of these systems to be determined. Several papers have already reported investigations of a phospholipid vesicle or ceil shape in an electric field. Shapes close to spherical have been investigated theoretically for the cases of constant [1] and alternating [2] electric fields. These studies were carried out assuming that the area of the cell membrane is constant and the analysis was performed for small deformations. Small changes of the membrane area were taken into account by Bryant and Wolfe [3], who analyzed the case of an a.c.electric field for an initially spherical cell.

The strict formalism describing the vesicle shape should be based on the condition that the local equilib-

\footnotetext{
- On leave from the A.N. Frumkin Institute of Electrochemistry. Russian Academy of Sciences. Moscow, Russian Federation.

** To whom correspondence should be addressed.
}

rium of each surface element is subject to the action of electrical and mechanical forces. The exact solution of this problem can be obtained by a numerical iterative procedure [4]. Such a procedure is cumbersome and therefore it is convenient to seek an adequate approximate solution of the problem. The aim of this paper is, first, the theoretical analysis of a large deformation of a vesicle in an electric field by taking into account the bending elastic properties of the membrane and parametrizing the shape in such a way that it is possible to determine the electrical forces analytically. For this reason the shape of the vesicle is assumed to be a rotational ellipsoid. The shape obtained by a small deformation of a sphere always belongs to this set and it is believed that they represent a good approximation at least for those volumes that do not deviate too much from the volume of a sphere. The second aim of this paper is to elucidate the corresponding dynamic behaviour of the system by including possible water transport through the membrane.

\section{Spheroidal static vesicle deformation}

The vesicle should have an axisymmetric shape and a centre of symmetry. In order to calculate electrical forces it is necessary to determine the electric field 
outside the vesicle. This problem can be solved analytically only for a very limited set of surfaces. We shall simplify the problem significantly by assuming that the shape of our vesicle belongs to that set. We choose the family of extended rotational ellipsoids (spheroids) with constant membrane area.

Let us introduce some notation. We shall be using the cylindrical coordinates $\mathrm{y}, \varphi$, $\mathrm{x}$ with the symmetry axis $\mathrm{x}$ oriented along the vector of external field strength $\boldsymbol{E}$,. The elongated spheroid (i.e. the ellipsoid, which is the body obtained by revolution of an ellipse) is then described by the equation

$y = b \longdiv { 1 - x ^ { 2 } / a ^ { 2 } }$

Here $a$ and $b$ are the parameters of the ellipsoid $(a>b)$. Owing to the condition of constant membrane area $S$, only one of them is independent [5]:

$S=4 \pi \int_{0}^{a} y \sqrt{1+(\mathrm{d} y / \mathrm{d} x)^{2}} \mathrm{~d} x$

$$
=2 \pi a^{2}\left(1-e^{2}+\sqrt{1 / e^{2}-1} \arcsin e\right)
$$

where e is the eccentricity of the ellipsoid and

$e^{2}=1-b^{2} / a^{2}$

In what follows we shall use the eccentricity e as an independent parameter, considering $\mathrm{a}$ and $\mathrm{b}$ as functions of e with the fixed parameter $S$. Once the membrane area is constant, eccentricity is the only parameter determining the volume of the ellipsoid:

$V=4 / 3 \pi a^{3}\left(1-e^{2}\right)$

We shall treat the mechanical aspects of the problem first and then the electrical aspects. The elastic energy of a flaccid vesicle is given by its membrane bending energy:

$W_{\mathrm{b}}=\frac{k_{\mathrm{c}}}{2} \oint H^{2} \mathrm{~d} S$

where $\mathrm{H}$ is the surface curvature, defined by its principal radii of curvature $R$, (meridian) and $R_{\mathrm{p}}$ (parallel),

$H=1 / R_{\mathrm{m}}+1 / R_{\mathrm{p}}$

and the constant $k_{\mathrm{c}}$ is the modulus of bending elasticity.

The expressions for the radius of curvature $R$, of the ellipse (cf. eqn. (1)) generating the ellipsoid is given by [5]

$R_{\mathrm{m}}=\left[\left(\frac{\mathrm{d} y}{\mathrm{~d} x}\right)^{2}+1\right]^{3 / 2}\left|\frac{\mathrm{d}^{2} y}{\mathrm{~d} x^{2}}\right|^{-1}$ or

$R_{\mathrm{m}}=a \frac{\left[1-(e x / a)^{2}\right]^{3 / 2}}{\sqrt{1-e^{2}}}$

According to Meneut's theorem for symmetricall surfaces $R,=y / \cos \theta$, where $\theta$ is the angle between the plane $\mathrm{x}=0$ and the normal to the surface at the point of interest. Because $\tan \theta=\mathrm{dy} / \mathrm{dx}$, the radius $R$, is expressed as follows:

$R_{\mathrm{p}}=a \sqrt{1-e^{2}} \sqrt{1-(e x / a)^{2}}$

The element $\mathrm{dS}$ of the surface of rotation can be expressed as

$\mathrm{d} S=y \sqrt{1+(\mathrm{d} y / \mathrm{d} x)^{2}} \mathrm{~d} x \mathrm{~d} \varphi$

We can substitute eqns. (6)-(9) into (5) and obtain the following expression for the membrane bending energy:

$W_{\mathrm{b}}=2 \pi k_{\mathrm{c}}\left(3-\frac{2 \mathrm{e}^{2}}{3}+\frac{\arcsin e}{e \sqrt{1-e^{2}}}\right)$

The dependence of $W_{\mathrm{b}}$ on e is shown in Fig. 1, curve 1. Differentiation of eqn. (10) with respect to e gives the variation of the bending elastic energy corresponding to the variation de of the eccentricity.

$\mathrm{d} W_{\mathrm{b}}$

$$
=8 \pi k_{\mathrm{c}}\left(-\frac{4 \mathrm{e}}{3}+\frac{\left.\mathrm{e}-1-2 \mathrm{e}^{2}\right) \arcsin e}{e^{2}\left(1-e^{2}\right)^{3 / 2}}\right) \mathrm{d} e
$$

The electrical forces involved arise as a consequence of putting the vesicle into a static field $E_{0}$. Here the vesicle is surrounded by a conducting medium and its

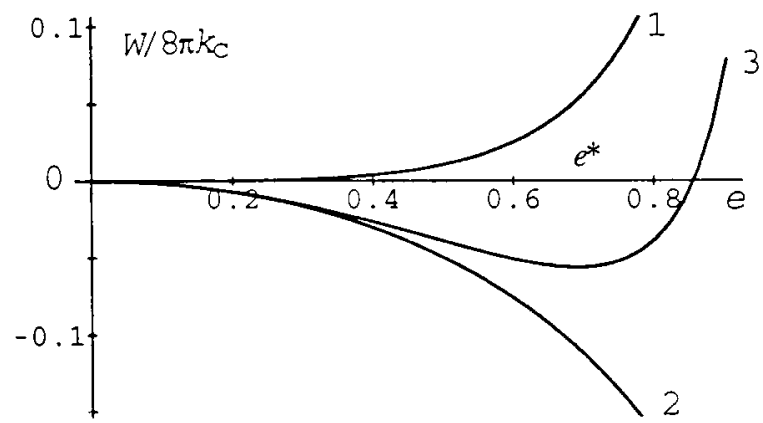

Fig. 1. The dependence of (1) the elastic bending energy $W_{\mathrm{b}}$, (2) the electrical energy $W_{\mathrm{el}}$ and (3) the total energy $W$ of the vesicle on the eccentricity e. All energies are given relative to the bending energy of a sphere $8 \pi k_{\mathrm{c}}$. Calculations of electrical energy are made for $\varepsilon=2.67$ 
inner content is also conducting. It is assumed that the membrane is non-conducting. Consequently, the field exists only outside the vesicle. Because the current density is proportional to the electric field strength (no concentration gradient), the problem is reduced to solving the Laplace equation for the electrical potential $\phi$ outside the vesicle:

$\Delta \phi=0$

At an infinite distance from the vesicle we have

$-\nabla \phi=\mathbf{E}_{0}$

where $\boldsymbol{E}_{0}$ is the external field strength. At the vesicle surface the normal component of the field strength must be zero:

$($ En $)=0$

where $\mathbf{n}$ is an externally oriented unit vector, normal to the surface. Equations (13) and (14) define necessary boundary conditions.

Let us consider the variation $\delta W_{\mathrm{el}}$ in electrical free energy of the system, defined as the work of the external forces which compensate the electrical forces for an infinitely small displacement $\delta \mathbf{u}$ of the elements of the surface:

$\delta W_{\mathrm{el}}=-\oint \boldsymbol{T} \delta \boldsymbol{u} \mathrm{d} S$

where $T$ is the electrical force per unit of the surface area. It is equal to the tensor product of the Maxwell stress tensor and the normal to the surface $\mathbf{n}[6]$ :

$\boldsymbol{T}=\boldsymbol{\epsilon} \epsilon_{0}\left[\mathbf{E}(\mathbf{E n})-\mathbf{n} E^{2} / 2\right]$

According to Eq. (14) the first term is zero, and as $T$ is normal to the vesicle surface, only normal components of $\mathbf{u}$ contribute to $\delta W_{\mathrm{el}}$, i.e.

$\delta W_{\mathrm{el}}=-\oint T \delta u_{\mathrm{n}} \mathrm{d} S$

Solution of the problem (12)-(14) is well known and can be found in the literature [6,7]. Transformation to the spheroidal coordinates is done in accordance with the formulae

$x=c \lambda \mu$

$y=c \sqrt{\lambda^{2}-1} \sqrt{1-\mu^{2}}$

where $\lambda \leq 1,0 \leq \mu \leq 1$ and $c=\sqrt{a^{2}-b^{2}}$ is the halfdistance between the ellipsoid foci. In the spheroidal coordinate system the Laplace equation (12) takes the form

$\frac{\partial}{\partial \lambda}\left[\left(\lambda^{2}-1\right) \frac{\partial \phi}{\partial \lambda}\right]+\frac{\partial}{\partial \mu}\left[\left(1-\mu^{2}\right) \frac{\partial \phi}{\partial \mu}\right]=0$

and the boundary conditions (13) and (14) become

$\phi \longrightarrow-E_{0} c \lambda \mu \quad$ at $\lambda \longrightarrow \infty$

$\partial \phi / \partial \lambda=0$

at $\lambda=1 / e$
The solution of problem (19)-(21) is

$\phi=-E_{0} c \mu\left[\lambda-\frac{Q(\lambda)}{Q^{\prime}(1 / e)}\right]$

where $Q(\lambda)$ is the first Legendre function of the second kind

$Q(\lambda)=\frac{\lambda}{2} \ln \left(\frac{\lambda+1}{\lambda-1}\right)-1$

and $Q^{\prime}$ is the derivative of $Q(\lambda)$ with respect to its argument. The value of the electric field $E$ on the surface is

$E=-\frac{\partial \phi}{\partial \mathbf{n}}=-\frac{1}{h} \frac{\partial \phi}{\partial \mu}$

where

$h=c \sqrt{\frac{\lambda^{2}-\mu^{2}}{1-\mu^{2}}}$

is the Lamé coefficient [5]. The value of the electrical field at the vesicle surface is expressed as

$E^{2}=\frac{E_{0}^{2}}{(1-f)^{2}} \frac{a^{2}-x^{2}}{a^{2}-e^{2} x^{2}}$

where

$f=\frac{1-e^{2}}{2 e^{3}}\left[\ln \left(\frac{1+e}{1-e}\right)-2 e\right]$

is the depolarization coefficient.

Let us now consider in detail the surface movement due to changing eccentricity. A small normal displacement $\delta u_{\mathrm{n}}$ of the vesicle surface corresponding to a small change $\mathrm{d} e$ in eccentricity is given by

$\delta u_{\mathrm{n}}=\left[\left(\frac{\mathrm{d} y}{\mathrm{~d} x}\right)^{2}+1\right]^{-1 / 2} \frac{\mathrm{d} y}{\mathrm{~d} e} \mathrm{~d} e$

In terms of Eqns. (1) and (3) we obtain

$\frac{\mathrm{d}\left(y^{2}\right)}{\mathrm{d} e}=\left(1-e^{2}\right) \frac{\mathrm{d}\left(a^{2}\right)}{\mathrm{d} e}-\left(a^{2}-x^{2}\right) 2 e$

The derivatives $\mathrm{d}\left(a^{2}\right) / \mathrm{d} e$ can be found by differentiation of (2):

$\frac{\mathrm{d}\left(a^{2}\right)}{\mathrm{d} e}=\frac{2 \pi a^{4}}{S}\left(2 e-\frac{1}{e}+\frac{\arcsin e}{e^{2} \sqrt{1-e^{2}}}\right)$

Because of the identity $\mathrm{d} y / \mathrm{d} e=(2 y)^{-1} \mathrm{~d}\left(y^{2}\right) / \mathrm{d} e$, eqn. (28) allows us to obtain $\delta u_{\mathrm{n}}$ after substitution of (1), (2), (29) and (30).

Then, after integration of eqn. (17), we obtain

$\mathrm{d} W_{\mathrm{el}}=\pi \epsilon \epsilon_{0} E_{0}^{2} a^{3}\left(e^{-2}-1\right)(1-f)^{-2} I \mathrm{~d} e$ 
where $\mathbf{I}$ is a dimensionless quantity depending on e:

$$
\begin{aligned}
I= & \left(e^{-2}-1\right)\left[1+\frac{e}{2 a^{2}} \frac{\mathrm{d}\left(a^{2}\right)}{\mathrm{d} e}\right] \ln \left(\frac{1+e}{1-e}\right) \\
& +a^{-2} \frac{\mathrm{d}\left(a^{2}\right)}{\mathrm{d} e}-\frac{4 e}{3\left(1-e^{2}\right)}-\frac{2}{e}
\end{aligned}
$$

In order to find the final expression for $W_{\mathrm{el}}$ we have to integrate (31) with respect to e:

$W_{\mathrm{el}}=\mathrm{d} W_{\mathrm{el}}=\int \frac{\mathrm{d} W_{\mathrm{el}}}{\mathrm{d} e} \mathrm{~d} e$

Combining results (11) and (31), we can calculate the variation in the free energy of the system, or the total work $\mathrm{d} W$ performed by external forces on the system at a small variation of the eccentricity de:

$\mathrm{d} W=\mathrm{d} W_{\mathrm{el}}+\mathrm{d} W_{\mathrm{b}}$

Figure 1 shows the dependence of $\mathrm{W}$ and its components $W_{\mathrm{el}}$ and $W_{\mathrm{b}}$ on eccentricity e.

It is possible to introduce a generalized force $\mathrm{F}$, acting along the e axis:

$F=-\frac{\mathrm{d} W}{\mathrm{~d} e}$

Solution of the equation $\mathbf{F}=0$ will correspond to an equilibrium shape of the vesicle. The dependence $F(e)$ is shown in Fig. 2. As $\mathbf{F}=0$ at $\mathrm{e}=0$, the point $\mathrm{e}=0$ is also an equilibrium point, although a non-stable one. The stable equilibrium of the system is established at a certain value of eccentricity which will be denoted by $\mathrm{e}^{*}$.

The parameters $E_{0}, \mathrm{~S}$ and $k_{r}$ enter the equation $\mathbf{F}=0$ in a single dimensionless combination:

$\mathscr{E}=E_{0}\left(\frac{S}{4 \pi}\right)^{3 / 4} \sqrt{\frac{\epsilon \epsilon_{0}}{k_{\mathrm{c}}}}$

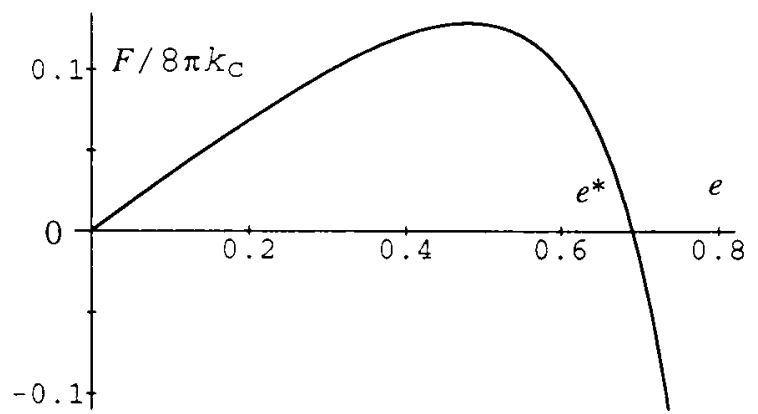

Fig. 2. The dependence of the generalized force $F$ (relative to the bending energy $8 \pi k c$ of a sphere) on the eccentricity $e$. The equilibrium exentricity is denoted by $\mathrm{e}^{*}$. The dimensionless electrical field strength is $\mathscr{E}=2.67$.

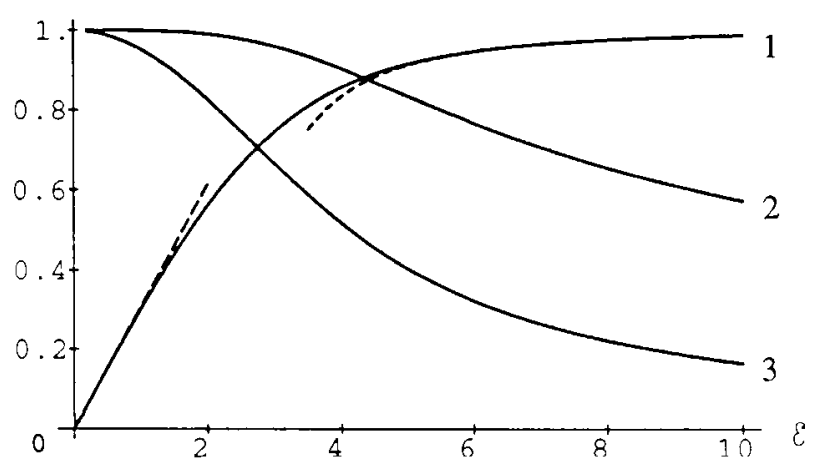

Fig. 3. The dependence of (1) the equilibrium excentricity e*, (2) the relative equilibrium vesicle volume $V / V_{0}$ and (3) the equilibrium semi-axes ratio $(b / a)^{*}$ on the external dimensionless electrical field strength $\mathscr{E}$. The broken lines correspond to the asymptotic expressions (45) and (46).

We can consider the quantity $\mathscr{E}$ as a dimensionless electrical field strength. Figure 3 shows the dependences of the equilibrium excentricity $\mathrm{e}^{*}$, the ratio $b / a$ and the normalized vesicle volume $V / V_{0}$ on the electrical field strength $\mathscr{E}$. Here $V_{0}=S^{3 / 2} / 6 \sqrt{\pi}$ is the volume of a sphere with the area of the surface equal to that of the vesicle $S$.

\section{Kinetics of vesicle deformation}

Whenever condition $\mathbf{F}=0$ is not satisfied, water flow through the membrane is expected. The corresponding vesicle volume change can be expressed as

$\frac{\mathrm{d} V}{\mathrm{~d} t}=-\kappa S p$

where $\kappa S$ is the water permeability of the membrane and $p$ is a pressure difference.

We assume that the field was applied to a spherical vesicle at time $t=0$. As the electrical charges redistribute much faster than the vesicle changes its shape, we can state that there is a generalized force $\mathrm{F}$ acting on the vesicle, moving it from the initial state to $\mathrm{e}^{*}$. The work $\mathrm{d} W$ is equal to the change $-p \mathrm{~d} V$ in the internal energy of the vesicle:

$\mathrm{d} \mathrm{W}=-p \mathrm{~d} V$

The value of the pressure difference $p$ can be calculated from (36) and the definition of the generalized force. Thus we obtain

$p=-F \mathrm{~d} e / \mathrm{d} V$

Taking the derivative of the vesicle volume (4) gives

$\frac{\mathrm{d} V}{\mathrm{~d} e}=\frac{4}{3} \pi a^{3}\left[\frac{3}{a}\left(1-e^{2}\right) \frac{\mathrm{d} a}{\mathrm{~d} e}-2 e\right]$ 


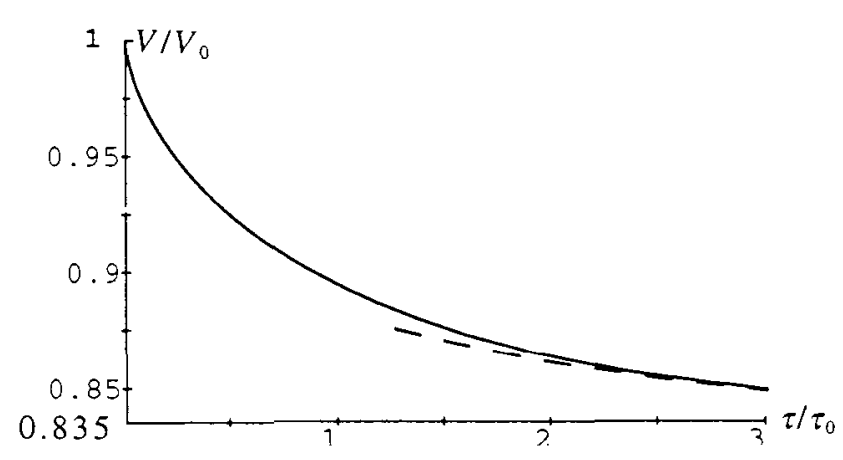

Fig. 4. Dependence of the vesicle volume on time $t / \tau_{0}$. The initial volume is the volume of the sphere. The final relative volume is $V / V_{0}=0.835$ which corresponds to the dimensionless electrical field $8=5$. The broken line shows the exponential relaxation.

Then, integrating eqn. (37), we obtain

$t=-\frac{1}{\kappa S} \int_{V_{0}}^{V} \frac{1}{F} \frac{\mathrm{d} V}{\mathrm{~d} e} \mathrm{~d} V=-\frac{1}{\kappa S} \int_{0}^{e} \frac{1}{F}\left(\frac{\mathrm{d} V}{\mathrm{~d} e}\right)^{2} \mathrm{~d} e$

This is an implicit expression for the eccentricity e, and therefore for the vesicle volume $\mathrm{V}$, as functions of time $t$ (Fig. 4).

Close to the equilibrium point the change in vesicle volume becomes a exponential with the characteristic time $\tau$ of the exponential relaxation:

$\tau=\left.\frac{1}{\kappa S}\left[\left(\frac{\mathrm{d} V}{\mathrm{~d} e}\right)^{2} / \frac{\mathrm{d} F}{\mathrm{~d} e}\right]\right|_{e=e^{*}}$

The right-hand side of eqn. (42) can be rewritten as a product of $\tau_{0}$, with the dimension of time, and the dimensionless combination:

$\tau=\left.\tau_{0}\left[\left(\frac{\mathrm{d} \nu}{\mathrm{d} e}\right)^{2} / \frac{\mathrm{d}\left(F / 8 \pi k_{\mathrm{c}}\right)}{\mathrm{d} e}\right]\right|_{e=e^{*}}$

where $\mathrm{v}$ is the relative volume $V / V_{0}$ and the proportionality factor $\tau_{0}$ is given by

$\tau_{0}=\frac{S^{2}}{288 \pi^{2} \kappa k_{\mathrm{c}}}$

The relaxation of vesicle volume and its exponential approximation are shown in Fig. 4

\section{Discussion}

We have suggested an approximate method of calculation of lipid vesicle shapes in an external homogeneous constant electrical field valid for relatively large vesicle deformations. The elastic properties of the vesicle are described by the bending elasticity modulus. The vesicle shape is approximated by a rotational ellipsoid. All the dimensional parameters of the problem are combined into a single dimensionless parameter $\mathscr{E}$ (eqn. (36)).

It can be proved by asymptotic analysis that, for a sufficiently long ellipsoid, the elastic forces are always greater than the electrical forces. Therefore the equilibrium solution exists for an arbitrary value of the electrical field. Simple analytical results can be obtained from the general equation $F=0$ for the extreme cases of weak and strong electrical fields. In the case of the weak electrical field we obtain

$e^{*}=\sqrt{\frac{3}{32}} \mathscr{E}$

which coincides with results obtained elsewhere [1,2]. For the strong fields we have

$e^{*}=1-\pi^{10 / 3} \frac{3^{4 / 3}}{32} \mathscr{E}^{-8 / 3}$

These simple formulae give results which deviate less than $20 \%$ from the exact solution of the equation $\mathrm{F}=0$ at $\mathrm{e}^{*}<0.5$ and $\mathrm{e}^{*}>0.9$ respectively (Fig. 3). Therefore they may be useful within the limits indicated for comparison with the experiment.

We shall indicate here the main practical application of the results obtained. Given the value of the experimentally measured eccentricity, we can solve the equation $\mathbf{F}=0$ and find the value of parameter $\mathscr{E}$. This enables us to estimate the bending elasticity modulus of the membrane.

Let us now discuss the kinetics of vesicle deformation. As has been shown above, the deformation kinetics is determined by the water permeability of the membrane. The characteristic time $\tau$ of this process has been calculated as a dependence on parameter $\mathscr{E}$ (Fig. 5(a))

For weak fields $\tau$ can be expressed as

$\frac{\tau}{\tau_{0}}=\frac{3 \pi}{2560} \mathscr{E}^{4}$

Comparison of this result with the exact one is shown in Fig. 5. The behaviour of $\tau(\mathscr{E})$, which is expressed in its non-monotonicity, is noteworthy. The most interesting is the increase of $\tau$ with $\mathscr{E}$, which is not immediately clear. However, this can be explained, if we note that the result (47) is valid for small $\mathscr{E}$ and therefore for small variations of the vesicle volume. In contrast, the volume change was expected to be comparable with the volume of the vesicle. The result obtained is important, because for nearly spherical vesicles the volume changes very slowly $\left(V / V_{0} \approx 1-\right.$ $\left.e^{4} / 15\right)$, although the dependence of the volume change on the eccentricity is very strong.

\footnotetext{
- This expression can easily be derived from eqn. (4) by substituting $a$ from eqn. (2).
} 
$W_{\mathrm{b}}$ elastic energy of vesicle, $\mathrm{J}$

$W_{\mathrm{el}}$ electrical energy of the system, $\mathrm{J}$

$\mathrm{x}$ axial coordinate, $\mathrm{m}$

y radial coordinate, $\mathrm{m}$

$\delta \boldsymbol{u}$ infinitely small displacement of the elements of the surface, $m$

$\Delta$ Laplace operator

$\epsilon$ coefficient of permittivity

$\epsilon_{0} \quad$ permittivity of free space, $8.8542 \times 10^{-12} \mathrm{~F} \mathrm{~m}^{-1}$

$\kappa \quad$ water permeability coefficient, $\mathrm{J}^{-1} \mathrm{~m}^{4} \mathrm{~s}^{-1}$

A spheroidal coordinate

$\mu$ spheroidal coordinate

$\theta$ angle between the plane $\mathrm{x}=0$ and the normal to the surface

$\tau$ characteristic time of the exponential relaxation, $\mathrm{s}$ $\tau_{0} \quad$ proportionality factor for $\tau, \mathrm{s}$
$\phi \quad$ electrical potential, $\mathrm{V}$
$\varphi$ angular coordinate
$\Gamma$ divergence operator

\section{References}

1 W. Helfrich. Z. Naturforsch.. c29 (1974) 182.

2 M. Winterhalter and W. Helfrich. J. Coll. Int. Sci.. 122 (1988) 583.

3 G. Bryant and J. Wolfe. J. Membr. Biol.. 96 (1987) 129.

3 B. Zeks. S. Svetina and V. Pastushenko. Biol. Membr.. 8 (1991) 429.

5 G. Korn and T. Korn, Mathematical Handbook. McGraw-Hill, New York, 1961. Chapter 6.

6 L.D. Landau and E.M. Lifshiz Electrodynamics of Continuous Media (2nd edn). Pergamon Press, Oxford. 1984.

7 H. Lamb. Hydrodynamics (6th edn.), Dover Publications. New York. 1945. 